This is an Accepted Manuscript version of the following article, accepted for publication in Journal of Affective Disorders. Xiaolu Zhou, Jessica Dere, Xiongzhao Zhu, Shuqiao Yao, Yulia E. Chentsova-Dutton \& Andrew G. Ryder (2011): Anxiety symptom presentations in Han Chinese and Euro-Canadian outpatients: Is distress always somatized in China?, Journal of Affective Disorders, https://doi.org/10.1016/j.jad.2011.06.049. (C)2011 Elsevier B.V. All rights reserved. This manuscript version is made available under the CC-BY-NC-ND 4.0 license https://creativecommons.org/licenses/by-nc-nd/4.0/

(@) $\Theta \Theta$

\title{
Anxiety symptom presentations in Han Chinese and Euro-Canadian outpatients: Is distress always somatized in China?
}

\author{
Xiaolu Zhou ${ }^{a}$, Jessica Dere ${ }^{b}$, Xiongzhao Zhuc, Shuqiao Yao ${ }^{c}$, Yulia E. Chentsova-Dutton ${ }^{d}$, Andrew G. \\ $\operatorname{Ryder}^{b, e}$, and * \\ ${ }^{a}$ Hunan Normal University \\ ${ }^{b}$ Concordia University \\ ${ }^{c}$ Central South University \\ ${ }^{d}$ Georgetown University \\ ${ }^{e}$ Sir Mortimer B. Davis - Jewish General Hospital
}

\begin{abstract}
Background: Cultural variations in the relative emphasis on somatic versus psychological symptoms of distress are a common topic in cultural psychopathology. The most well-known example involves people of Chinese heritage, who are found to emphasize somatic symptoms in presenting depression as compared with people of Western European heritage. It remains unknown whether a similar cultural difference is found for anxiety disorders.

Methods: Euro-Canadian $(n=79)$ and Han Chinese $(n=154)$ psychiatric outpatients with clinically significant concerns about both depression and anxiety were selected from a larger dataset based on their responses to a structured interview. They also completed two self-report questionnaires assessing somatization of depression and anxiety.

Results: As expected, Chinese participants reported a greater tendency to emphasize somatic symptoms of depression, as compared to the Euro-Canadians. Contrary to expectations, the tendency to emphasize somatic symptoms of anxiety was higher among the Euro-Canadians as compared to the Chinese participants.

Limitations: Characteristics of our participants limit the generalizability of our findings. The current study is preliminary and requires replication.

Conclusions: Despite the exploratory nature of this study, the results suggest that the popular notion of 'Chinese somatization' should not be over-generalized. Our findings also imply that there may be important differences in the cultural understanding of depression and anxiety in both Chinese and 'Western' contexts. Future studies should seek to unpack potential cultural explanations for why Euro-Canadian outpatients may emphasize somatic symptoms in the presentation of anxiety to a greater degree than Chinese outpatients.

(C)2011 Elsevier B.V. All rights reserved.
\end{abstract}

Keywords: Anxitety,Depression, Somatization, Culture, Chinese, Euro-Canadian

\section{Introduction}

Among the most well-known examples of culture shaping psychopathology is the extent to which distressed people in different cultural contexts emphasize somatic symptoms in the presence of psychosocial difficulties (Kirmayer and

Even without long term worries, there must be immediate concerns. (King of Weiling, the Analects of Confucius)

* Corresponding author at: Department of Psychology (PY1532), Concordia University, 7141 rue Sherbrooke O., Montréal, Québec H4B1R6, Canada. Tel.: +1 5148482424 x 5379; fax: +1 514848 4523. E-mail address: andrew.ryder@concordia.ca (A.G.Ryder).
Robbins, 1991; Kleinman, 1982, 1986). Although at least some degree of somatic symptom emphasis - or 'somatization' - is found worldwide, considerable variation is observed across cultural contexts (Simon et al., 1999; Weissman et al., 1996). Failure by clinicians to recognize predominantly somatic presentations as connected to anxiety or depression may lead to misdiagnosis, and hence to inadequate or inappropriate treatment (Kirmayer, 2001).

The most frequently cited example involves people of Chinese heritage, described as having a strong tendency to emphasize somatic symptoms compared with people of Western European heritage. This difference has been confirmed with respect to depression symptoms in two direct crossgroup comparisons involving clinical outpatients (Parker et al., 2001; Ryder et al., 2008). Many culturally-based explanations have been proposed to explain this cultural vari- 
ation, most of which posit that somatic versus psychological experiences differ either in terms of their importance or their acceptability (Ryder et al.,2002). Although this group difference has received considerable attention, the question of whether a similar difference can be found among anxiety disorders remains unexplored.

As with depression, anxiety disorders are defined by a blend of psychological (e.g., worry, difficulty concentrating) and somatic (e.g., tension, sweating) symptoms. Ryder et al. (in press) have described ways in which cultural scripts for different forms of distress can shape which symptoms are experienced and expressed to others. These scripts include both beliefs about the meaning of symptoms (e.g., important others will feel like I am judging them if I tell them I feel hopeless about the future) and concrete behavioral practices (e.g., recruiting help from important others by emphasizing sleep and digestive problems). Lacking much previous research, however, it is difficult to make strong predictions about the cultural scripts operating for anxiety disorders, and hence what variations in symptom presentations to expect.

On the one hand, we might expect a similar cultural variation in anxiety as observed with depression: a tendency for Chinese people with anxiety disorders to emphasize somatic symptoms. To begin with, both disorders are characterized by negative affect, and hence there is considerable overlap between them. Neuroimaging work suggests that anxiety and depression are associated with similar interpretation biases for interoceptive information (Paulus and Stein, 2010), which could lead to a common consequence - somatic symptom expression. If more somatic symptom reporting is observed in Chinese populations due to a cultural script for the presentation of negative affect, we would expect this effect to generalize across disorders in which negative affect is prominent. Reviews of Traditional Chinese Medicine provide some support for this view - here, somatic symptoms are seen as aspects of emotions and are embedded in Chinese daily life (Hong et al., 1995; Lin, 1985; Tseng, 1975).

On the other hand, cultural scripts for different forms of distress are closely related to the meaning that syndromes and their symptoms have in a given cultural context; depression and anxiety may carry different meanings. Chinese cultural contexts foster a strong prevention focus, an orientation towards avoiding negative outcomes that is closely tied to obligations towards others (Elliot et al., 2001; Lee et al., 2000; Lockwood et al., 2002) and that is also linked to anxiety-related emotions (Higgins et al., 1997; Lee et al., 2000). Anxiety may therefore be more acceptable than depression in Chinese contexts, in that anxiety communicates sensitivity to social cues. The somatic symptoms of anxiety may also be less effective in expressing these prosocial norms as compared with the psychological symptoms, which contain much of the specific content (e.g., worry about friends, obsessive thinking about family's safety). Following this ar- gument, we would expect similar levels of somatic symptoms between Chinese and Euro-Canadian patients.

Little research has focused on anxiety disorder symptoms among outpatient samples of Chinese and Western European heritage. The current study represents an initial attempt to examine how culture shapes the self-reported tendency among patients to emphasize somatic symptoms when anxious. Specifically, the study seeks to compare the two competing predictions described above and begin addressing whether 'Chinese somatization' is specific to depression or also observed in anxiety.

\section{Method}

\subsection{Participants}

Participants in the current study were selected from a larger sample described by Ryder et al. (2008). That study contains detailed information about the research sites in Toronto (Canada) and Changsha (China), recruitment, inclusion and exclusion criteria, study measures, and sample equivalence. The study received ethical approval at all involved institutions, and was conducted in accordance with the Helsinki Declaration, 1989 revision; all participants provided informed consent. For the current study, participants' responses on a structured interview were used to select patients that had at least one clinically significant anxiety concern (i.e., panic attacks, agoraphobic anxiety, social anxiety, worry, obsessions, compulsions, or phobic anxiety; PTSD was not assessed). The final Toronto sample included 33 men and 46 women of Euro-Canadian heritage, with a mean age of 36 years (range $=18$ to $60, S D=10$ ); the final Changsha sample included 68 men and 86women of Han Chinese heritage, with a mean age of 31 years (range $=18$ to $65, S D=$ 11).

\subsection{Interview}

All participants were assessed with the Structured Clinical Interview for DSM-IV, Axis I, Patient Version, anxiety disorder modules (First et al., 1997). Ryder et al. (2008) describe several modifications made to the interview, including use of a 0 to 3 rating scale. Anxiety concerns, defined as the core symptom(s) of each type of anxiety disorder (e.g., panic attack in Panic Disorder, obsessions and compulsions in OCD) were used for participant selection. Following Ryder et al. (2008), formal diagnoses were not used as diagnosis itself is shaped by culture and, moreover, insistence on strict diagnostic categories can serve to artificially reduce cultural variation (Kleinman, 1988). 


\subsection{Questionnaires}

\subsubsection{CPAI-Somatization (CPAI-S)}

The somatization subscale of the Chinese Personality Assessment Inventory (CPAI-S; Cheung et al., 1996) is a 15item measure of attitudes, beliefs, and physical responses to psychosocial stressors thought to increase the likelihood of emphasizing somatic symptoms when depressed. A sample item is, "I get a headache every time I am annoyed". Internal consistency coefficients were .62 in Toronto and .73 in Changsha.

\subsubsection{Cognitive-Somatic Anxiety Questionnaire (CSAQ)}

The CSAQ (DeGood and Tait, 1987) consists of two 7item subscales. Items begin with the stem, "When I feel anxious, nervous, or tense..." Items on the somatic subscale (CSAQ-S) assess the general tendency towards somatic symptoms when anxious; a sample item is, "... my heart beats faster". Internal consistency coefficients were .58 in Toronto and .66 in Changsha. Items on the cognitive subscale (CSAQ-C) assess the tendency towards cognitive symptoms when anxious; a sample item is, "...I imagine terrifying scenes". Internal consistency coefficients were .76 in both Toronto and Changsha

\section{Results}

\subsection{Cross-cultural equivalence}

Ryder et al. (2008) provide a detailed description of the methods used for the assessment of structural and item equivalence. Three-step ordinal logistic regressions did not identify any items with differential item functioning on the CPAIS, CSAQ-S, or CSAQ-C.

\subsection{Group comparisons on anxiety concerns}

Levels of anxiety concerns in each group are shown in Table 1, along with group comparisons. Participants in the Toronto sample reported significantly higher levels of panic attacks and social anxiety, while those in the Changsha sample reported significantly higher levels of worry and obsessions.

\subsection{Group comparisons on somatization tendency}

Table 1 displays group means on the self-report measures, along with group comparisons. Scores on the CPAI-S, CSAQ-S, and CSAQ-C were all significantly correlated with one another within each group, $r s=.32$ to .60 , all $p s<.01$. Therefore, ANCOVA was used to compare the groups on the somatization scales, controlling for the other scales. Consistent with Ryder et al. (2008), the tendency to somatize depression (CPAI-S) was significantly higher in Changsha than in Toronto after controlling for the CSAQ-S and CSAQ-C,
$F(1,228)=33.58, p<.01$. However, the tendency to somatize anxiety (CSAQ-S) was significantly higher in Toronto than in Changsha after controlling for the CSAQ-C and CPAI-S, F( $(1,228)=70.00, p<.01$.

\section{Discussion}

Due to the lack of previous cross-cultural research on somatic symptom presentations in anxiety disorders, we began this study with two rival predictions: (1) that Chinese psychiatric outpatients would report a greater tendency to present somatic symptoms when anxious compared to EuroCanadians; or (2) no such difference. Instead, we found that Chinese outpatients endorsed a tendency to present fewer somatic symptoms when anxious compared to Euro-Canadians. What accounts for this unexpected finding?

We have already discussed cultural variations in regulatory orientations as reason to expect that the cultural shaping of anxiety symptoms does not necessarily mirror that of depressive symptoms. A prevention focus tends to be more strongly endorsed in Chinese contexts, linking prosocial concerns with greater anxiety. Even if this explanation is supported by future research, however, we still must account for greater endorsement of a tendency to present anxiety somatically among Euro-Canadian compared with Chinese psychiatric outpatients. One possibility is that in cultural contexts with low levels of prevention focus there is little personal or social value in psychological symptoms of anxiety, and so the cultural script focuses attention on the somatic experience instead. It is also possible, however, to go further and propose that anxiety specifically violates cultural expectations common to North American contexts. In settings where one is expected to confidently pursue one's own goals and present oneself positively to others, anxiety may be particularly problematic to the extent that it directly interferes with these expectations.

One limitation of this study is that it looks at self-reported tendency to present certain kinds of symptoms when anxious, rather than current anxiety symptoms. This approach was necessary in this study because specific anxiety questions were only posed to patients who endorsed a particular anxiety concern (e.g., only patients who endorsed significant worry were asked the remaining questions pertinent to Generalized Anxiety Disorder). Nonetheless, the most pronounced group differences on current anxiety concerns are consistent with our main findings: the Euro-Canadian sample had higher rates of panic attacks, a markedly somatic form of anxiety (see also Lee et al., 2005); the Chinese sample, meanwhile, had higher rates of obsessive thinking. Several other concerns are not easily categorized as somatic or psychological, however, making it difficult to rely on current symptoms to compare our predictions.

There are several additional limitations that should be acknowledged. First, the CSAQ-S showed low reliability in 
Table 1

Summary of descriptive statistics and group differences.

\begin{tabular}{|c|c|c|c|c|c|c|}
\hline & \multicolumn{2}{|c|}{ Toronto } & \multicolumn{2}{|c|}{ Changsha } & \multicolumn{2}{|c|}{$t^{\prime}$ test $^{\mathrm{a}}$} \\
\hline & M & SD & M & SD & $t^{\prime}$ & df \\
\hline Panic attacks & 0.72 & 0.96 & 0.25 & 0.67 & $3.93^{* *}$ & 118.06 \\
\hline Agoraphobic anxiety & 0.38 & 0.79 & 0.31 & 0.72 & 0.64 & 145.07 \\
\hline Social anxiety & 0.85 & 1.04 & 0.49 & 0.83 & $2.68^{* *}$ & 130.84 \\
\hline Worry & 1.35 & 1.05 & 1.74 & 0.98 & $-2.72^{* *}$ & 147.56 \\
\hline Obsessions & 0.19 & 0.51 & 0.86 & 1.08 & $-6.40^{* *}$ & 229.63 \\
\hline Compulsions & 0.37 & 0.68 & 0.31 & 0.76 & 0.56 & 173.61 \\
\hline Phobic anxiety & 0.59 & 0.87 & 0.59 & 0.98 & 0.03 & 174.98 \\
\hline CPAI-S & 2.70 & 0.47 & 2.92 & 0.57 & $-3.11^{* *}$ & 186.64 \\
\hline CSAQ-S & 3.40 & 0.61 & 2.72 & 0.69 & $7.63^{* *}$ & 171.59 \\
\hline CSAQ-C & 3.68 & 0.67 & 3.46 & 0.73 & $2.28^{*}$ & 166.16 \\
\hline
\end{tabular}

Note. CPAI-S = Chinese Personality Assessment Inventory-Somatization; CSAQ-S = Cognitive-Somatic Anxiety Questionnaire-Somatic subscale; CSAQ -C Cognitive-Somatic Anxiety Questionnaire-Cognitive subscale. aGiven the unequal group sizes, Welch's t-test (i.e., $t^{\prime}$ ) was used in place of the regular $\mathrm{t}$ test, to account for potential differences in variance.

$* * p<.01$.

$* p<.05$.

the Toronto sample, indicating a need for improved measurement in future studies. In addition, participants at both sites sought help at a major urban psychiatric center, limiting the generalizability of our findings. In particular, it is likely that the Chinese participants would be considerably more 'Westernized' than other Chinese groups, such as migrant workers or rural patients (Ryder et al., 2008). Finally, this study examined anxiety within a depressed sample, and lacked sample size for examination of specific anxiety disorders. However, in light of the high levels of comorbidity between depression and the various anxiety disorders, the diagnostic heterogeneity of our sample reflects clinical reality.

The current study is the first effort, to our knowledge, to extend cultural comparative research on somatic symptom presentations to anxiety disorders. Discussions of 'Chinese somatization', although based largely on clinical observations and studies of depressed patients, often describe somatic symptom emphasis as a general tendency in Chinese populations. These results suggest instead that this finding is specific to depression and should not be over-applied; they point to ways in which cultural scripts may differ for anxiety and depression, raising possible explanations for our findings that await direct empirical investigation in future studies. Unexpected evidence for 'Western somatization' of anxiety serves as a reminder that Western contexts should not be taken as the norm from which other contexts differ, but rather are themselves specific milieus that merit cultural explication.

\section{Role of funding source}

Preparation of this manuscript was supported by a New Investigator Award to AGR from the Canadian Institutes of Health Research. CIHR had no further role in: study design; collection, analysis, and interpretation of data; writing the report; or the decision to submit for publication. 


\section{Conflict of interest}

The authors declare that none of them have any conflicts of interest.

\section{Acknowledgment}

We acknowledge the contributions of the collaborators, translators, and interviewers who contributed to the original study — Jason Bacchiochi, Mike Bagby, Niki Fitzgerald, Steve Heine, Janice Mak, Lesley Miller, Kei-Yan Poon, Tina Wang, Maria Wong, Jian Yang, and Jinyao Yi. We are also grateful to Ying Wong for her assistance in thinking through the cultural explanations proposed in this paper.

\section{References}

Cheung, F.M., Leung, K., Fan, R., Song, W.Z., Zhang, J.X., Zhang, J.P., 1996. Development of the Chinese Personality Assessment Inventory (CPAI). J. Cross. Cult. Psychol. 27, 181-199.

DeGood, D.E., Tait, R.C., 1987. The Cognitive-Somatic Anxiety Questionnaire: psychometric and validity data. J. Psychopathol. Behav. 9, 75-87.

Elliot, A.J., Chirkov, V.I., Kim, Y., Sheldon, K.M., 2001. A cross-cultural analysis of avoidance (relative to approach) personal goals. Psychol. Sci. 12, 505-510.

First, M.B., Spitzer, R.L., Gibbon, M., Williams, J.B.W., 1997. Structured Clinical Interview for DSM-IV Axis I Disorders, Research Version, Patient Edition (SCID-I/P). Biometrics Research. New York State Psychiatric Institute, New York, NY.

Higgins, E.T., Shah, J., Friedman, R., 1997. Emotional responses to goal attainment: strength of regulatory focus as a moderator. J. Pers. Soc. Psychol. 72, 515-525.

Hong, G.K., Lee, B.S., Lorenzo, M.K., 1995. Somatization in Chinese American clients: implications for psychotherapeutic services. J. Contemp. Psychother. 25, 105-118.

Kirmayer, L.J., 2001. Cultural variations in the clinical presentation of depression and anxiety: implications for diagnosis and treatment. J. Clin. Psychiatry 62, 22-28.

Kirmayer, L.J., Robbins, J.M., 1991. Introduction: concepts of somatization. In: Kirmayer, L.J., Robbins, J.M. (Eds.), Current Concepts of Somatization: Research and Clinical Perspectives. American Psychiatric Press, DC, pp. $1-19$.

Kleinman, A., 1982. Neurasthenia and depression: a study of somatization and culture in China. Cult. Med. Psychiatry 6, 117-190.

Kleinman, A., 1986. Social Origins of Disease and Distress: Depression, Neurasthenia, and Pain in Modern China. Yale University Press, New Haven, CT.
Kleinman, A., 1988. Rethinking Psychiatry: From Cultural Category to Personal Experience. The Free Press, New York, NY.

Lee, A.Y., Aaker, J.L., Gardner, W.L., 2000. The pleasures and pains of distinct self-construals: the role of interdependence in regulatory focus. J. Pers. Soc. Psychol. 78, 1122-1134.

Lee, S., Tsang, A., Kwok, K., 2005. A community-based telephone survey of panic disorder in Hong Kong. Depress. Anxiety 22, 77-83.

Lin, T.Y., 1985. Mental disorders and psychiatry in Chinese culture: characteristic features and major issues. In: Tseng, W.S., Wu, D.Y.H. (Eds.), Chinese Culture and Mental Health. Academic Press, NY, pp. 369-394.

Lockwood, P., Jordan, C.H., Kunda, Z., 2002. Motivation by positive or negative role models: regulatory focus determines who will best inspire us. J. Pers. Soc. Psychol. 83, 854-864.

Parker, G., Cheah, Y.C., Roy, K., 2001. Do the Chinese somatize depression? A cross-cultural study. Soc. Psychiatry Psychiatr. Epidemiol. 36, 287-293.

Paulus, M.P., Stein, M.B., 2010. Interoception in anxiety and depression. Brain Struct. Funct. 214, 451-463.

Ryder, A.G., Yang, J., Heine, S.J., 2002. Somatization vs. psychologization of emotional distress: a paradigmatic example for cultural psychopathology. In: Lonner, W.J., Dinnel, D.L., Hayes, S.A., Sattler, D.N. (Eds.), Online Readings in Psychology and Culture. Western Washington University: Center for Cross-Cultural Research. Retrieved from www.wwu.edu/ culture.

Ryder, A.G., Yang, J., Zhu, X., Yao, S., Yi, J., Heine, S.J., Bagby, R.M., 2008. The cultural shaping of depression: somatic symptoms in China, psychological symptoms in North America? J. Abnorm. Psychol. 117, 300-313.

Ryder, A. G., Ban, L. M., Chentsova-Dutton, Y. E., in press. Towards a Cultural Clinical Psychology. Soc Pers Psychol Compass.

Simon, G.E., VonKorff, M., Piccinelli, M., Fullerton, C., Ormel, J., 1999. An international study of the relation between somatic symptoms and depression. N. Engl. J. Med. 341, 1329-1335.

Tseng, W.S., 1975. The nature of somatic complaints among psychiatric patients: the Chinese case. Compr. Psychiatry 16, 237-245.

Weissman, M.M., Bland, R.C., Canino, G.J., Faravelli, C., Greenwald, S., Hwu, H.-G., Joyuce, P.R., Karam, E.G., Lee, C.-K., Lellouch, J., Lépine, J.-P., Newman, S.C., Rubio-Stipec, M., Wells, E., Wickramaratne, P.J., Wittchen, H.-U., Yeh, E.-K., 1996. Cross-national epidemiology of major depression and bipolar disorder. J. Am. Med. Assoc. 276, 293-299. 